\author{
Associate Professor Jian-Zhang WU, PhD \\ E-mail: sjzwjz@gmail.com \\ School of Business, Ningbo University, Ningbo, China \\ Rui-Jie XI, MS Candidate \\ E-mail: jessie_xi921@163.com \\ School of Business, Ningbo University, Ningbo, China \\ Professor Gleb BELIAKOV, PhD \\ E-mail:gleb@deakin.edu.au \\ School of Information Technology \\ Deakin University, Melbourne, Australia
}

\title{
NONADDITIVITY INDEX ORIENTED DECISION PREFERENCE INFORMATION REPRESENTATION AND CAPACITY IDENTIFICATION
}

\begin{abstract}
The nonadditivity index is a competent indicator of depicting the kind and intensity of interaction among decision criteria. In this paper, we focus on using the nonadditivity index to represent the decision maker's preference information as well as the process of transforming them into standard capacity. We first discuss the comparison and range representation forms of decision preference information in terms of nonadditivity index and update the inconsistency recognition models and adjustment strategies. Then we establish a nonadditivity index oriented multiple goal linear programming algorithm to find out the minimum deviation capacities with relatively less concerns and efforts on inconsistency adjustment. The illustrative example demonstrates the feasibility and flexibility of the proposed scheme and methods.

Keywords: Fuzzy measure, Nonadditivity, Interaction index, Inconsistency, Capacity identification.
\end{abstract}

JEL Classification: C44, C61, C88, D83

\section{Introduction}

Capacity [5] is also called nonadditive measure [15,17] for its inherent nonadditivity property which corresponds to the additivity property of probability measure and meanwhile provides an explicit view of the interaction phenomenon among criteria. Generally speaking, additivity means the criteria are independent or with zero interaction; superadditivity means the criteria are complementary or with

DOI: 10.24818/18423264/54.2.20.17 
positive interaction; subadditivity means the criteria are substitutive or with negative interaction $[2,9]$.

As an indicator of directly depicting the kind and density of nonadditivity as well as of the associated interaction, nonadditivity index of capacity is proposed and discussed lately [18]. Compared to the traditional simultaneous interaction indices, the nonadditivity can reasonably describe the interaction among decision criteria and also has some good properties [20,21], such as the uniform range of different cardinalities, moderate compromise with the effect of dummy, extreme interaction property. Some applications of nonadditivity index in the context of capacity based decision making are introduced, see [19,22].

The capacity based decision maker's preference information on the importance and interaction of decision criteria are usually provided in terms of the comparison and interval forms $[1,10,12,16,24,25]$, e.g., the importance/interaction of a criteria subset $A$ is larger than that of $B$ with a threshold of $\delta$, and the importance/interaction belongs to an interval $[\alpha, \beta]$. Since the simultaneous interaction index has changing ranges for different cardinalities and confusing explanation of indices for 3 or more criteria in decision context, traditionally the preference information only apply for one criterion and two criteria $[2,8,9,13]$. However, by virtue of the uniform ranges and other mathematic properties of the nonadditivity index, these comparison and interval forms can be carried out for arbitrary cardinality subsets and the interaction degrees can be easily specified and corresponded to some regular ranges for all subsets as well [18,20,21].

It is inevitable that some inconsistencies exist among the decision maker's preference information since the inherent uncertainty and complexity within the decision problem [2]. In the scheme of capacity-based decision analysis, the inconsistency recognition and adjustment approaches are generally resorted to the 0-1 mixed linear programming model $[6,7]$ or the multiple goal linear programming (MGLP) model $[19,22]$. The two categories of approaches both are competent to recognize the inconsistent constraints without much field knowledge but the MGLP models can further provide some adjustment suggestions based on the recognition results. However, we found that the previous MGLP model basically only discuss the boundary inconsistency in some situation. In this paper, we further update the MGLP model to more reasonably recognize the contradiction among the whole domain of preference constraints, and provide some flexible adjustment strategies and models accordingly.

Furthermore, mainly inspired by the work of simultaneous interaction indices oriented capacity identification method given in [23], we also construct the nonadditivity index oriented capacity identification algorithm, which aims to use the coefficients in objective function of MGLP model to differentiate the ranges of the nonadditivity index and get the satisfied compromise result of all the variables. The major advantage of this algorithm is it can significantly reduce the efforts on recognizing and adjusting inconsistency by only remaining the comparison constraints of singletons' indices. 
Nonadditivity Index Oriented Decision Preference Information Representation and Capacity Identification

This paper is organized as follows. After the introduction, we briefly present background knowledge of the capacity and nonadditivity index in Section 2. In Section 3, we discuss the inconsistency recognition models and adjustment strategies. Section 4 is for the nonadditivity index oriented algorithm. In section 5, we use an illustrative example to present the proposed model and algorithm in detail. Finally, we conclude the paper in Section 6.

\section{Preliminaries}

Let $N=\{1,2, \ldots, \mathrm{n}\}, n>2$, be the decision criteria set, $P(N)$ be the power set of $N$, and $|S|$ be the cardinality of subset $S \subseteq N$.

Definition 1. $[5,12,17]$ A capacity on $N$ is a set function $\mu: P(N) \rightarrow[0,1]$ such that

(1) $\mu(\varnothing)=0, \mu(N)=1 ;$ (boundary condition)

(2) $\forall A, B \subseteq N, A \subseteq B$ implies $\mu(A) \leq \mu(B)$. (monotonicity condition)

The monotonicity condition of capacity reveals the essential property about decision criteria to the decision problem, i.e. the importance of a criteria subset cannot decrease when new criteria are added [3,12].One of the explicit characteristics stems from the monotonicity w.r.t. inclusion subsets is the nonadditivity w.r.t. disjoint subsets that leads to the alternative name of capacity, the nonadditive measure.

Definition 2. [18] A capacity $\mu$ on $N$ is said to be $\star$-additive within $S \subseteq N$, if $\mu(A \cup B) \stackrel{\star}{=} \mu(A)+\mu(B), \forall A, B \subseteq S, A, B \neq \emptyset, A \cap B=\emptyset$, where “ $\stackrel{\star}{=}$ stands for "= (resp. $\geq, \leq$, > and $<$ )", " $\star$-additive" stands for "additive (resp. superadditive, subadditive, strict superadditive, and strict subadditive)".

Generally speaking, superadditivity means the complementary or positive interactions of decision criteria while the subadditivity means the substitutive or negative interactions. The traditional simultaneous interaction index, like the Möbius representation [4] and the Shapley interaction indices [11], can not correctly reflect the above intuitive judgment, see some counterexamples in $[18,21]$. In view of this major shortcoming of simultaneous interaction index and its other inconveniences in application, e.g., the changing ranges of different cardinalities, we proposed nonadditivity index which has some good mathematical characteristics and intuitive interpretations [18].

Definition 3. [18] The nonadditivity index of a subset $A \subseteq N$ w.r.t. $\mu$ is defined as

$$
n_{\mu}(A)=\mu(A)-\frac{1}{2^{|A|-1}-1} \sum_{C \subset A} \mu(C) .
$$

Proposition 1. For a nonadditivity index $n_{\mu}$ of a $\mu$ on $N$,

(1) If $\mu$ is $\star$-additive within $S \subseteq N$, then $n_{\mu}(A) \stackrel{\star}{=} 0, \forall A \subseteq S,|A| \geq 2$;

(2) $-1 \leq n_{\mu}(A) \leq 1, \forall A \subseteq N$;

DOI: 10.24818/18423264/54.2.20.17 
Jian-Zhang Wu, Rui-JieXu, GlebBeliakov

(3) $n_{\mu}(A)=1 \Leftrightarrow \mu(B)=0, \forall B \subset A$, and $\mu(A)=1$;

(4) $n_{\mu}(A)=-1 \Leftrightarrow \mu(B)=1, \forall B \subseteq A$, and $B \neq \emptyset$;

The item (1) means that the signs of nonadditivity index are in accordance with the types of interactions. The item (2) show the ranges of nonadditivity indices are normalized into the uniform intervals $[-1,1]$, so different cardinality indices can be directly compared. The items (3) and (4) respectively give the positive and negative extreme interaction cases, which is also easy to understand in the context of decision making.

Similar to the Möbius representation [4] as well as the Shapley interaction index [14], the nonadditivity index is also a representation of capacity [18].

Proposition 2. A capacity $\mu$ on $N$ can be represented through nonadditivity index $n_{\mu}$ as

$$
\mu(A)=n_{\mu}(A)+\sum_{C \subset A} \sum_{i=|C|}^{|A|-1} \prod_{j=i}^{|A|-1} \frac{|A|-j}{2^{j}-1} n_{\mu}(C), \forall A \subseteq N .
$$

From the definition of capacity and the above proposition, we can have the following result $[19,20]$.

Proposition 3. The coefficients set $\left\{n_{\mu}(A)\right\}_{A \subseteq N}$ corresponds to the nonadditivity index of a capacity on $\mathrm{N}$ iff

(1) $n_{\mu}(\varnothing)=0, n_{\mu}(N)+\sum_{C \subset N} \sum_{i=|C|}^{n-1} \prod_{j=i}^{n-1} \frac{n-j}{2^{j}-1} n_{\mu}(C)=1$;

(2) $n_{\mu}(A \cup\{i\})+\sum_{C \subset A \cup\{i\}} \sum_{i=|C|}^{|A|} \prod_{j=i}^{|A|} \frac{|A|+1-j}{2^{j}-1} n_{\mu}(C)-n_{\mu}(A)$ $-\sum_{C \subset A} \sum_{i=|C|}^{|A|-1} \prod_{j=i}^{|A|-1} \frac{|A|-j}{2^{j}-1} n_{\mu}(C) \geq 0, \forall i \in N, A \subseteq N \backslash\{i\}$.

The above two propositions show the nonadditivity index, as a one-to-one mapping of capacity, can be applied as an alternative index to represent the capacity as well as its related conceptions and models.

\section{The preference representation and adjust strategies}

\subsection{The comparison and interval representation forms}

As mentioned in Introduction, the decision maker's preference information on the importance and interaction of decision criteria are usually provided in terms of the comparison and interval forms [12,13]. For the comparison form, we can directly compare the nonadditivity indices with different cardinalities, e.g., we can say the index of subset $\{1,2,3\}$ is greater than $\{1,2,3,4\}$ with threshold of 0.1 , which means the criteria 4 is likely substitutive with criteria 1,2 and 3 . For the interval range, we can divide the range of $[-1,1]$ into some sub intervals to show the density of interaction. For example, by equivalent divisions, we can just use the intervals

$$
[-1,-0.75),[-0.75,-0.5),[-0.5,-0.25) \text { and }[-0.25,0)
$$

to show the extremely, strongly, fairly and slightly negative interactions, and use

$$
[1,0.75),[0.75,0.5),[0.5,0.25) \text { and }[0.25,0)
$$


Nonadditivity Index Oriented Decision Preference Information Representation and Capacity Identification

to show the corresponding positive interactions, and the zero interaction just means the nonadditivity index is 0 . For convenience, we just call this range division form as the 9-interval interaction scale. Since the nonadditivity index is a liner representation of capacity, the decision maker's preference information with comparison and interval forms are essentially the linear constraints with respect to capacity $[1,2,25]$.

\subsection{The MGLP based inconsistency check method}

It is inevitable that some inconsistencies exist among the decision maker's preference information since the inherent uncertainty and complexity within the decision problem and environment as well.

One of the inconsistency check approaches is based on the multiple goal linear programming (MGLP) [2,19,22], see the Algorithm 1.

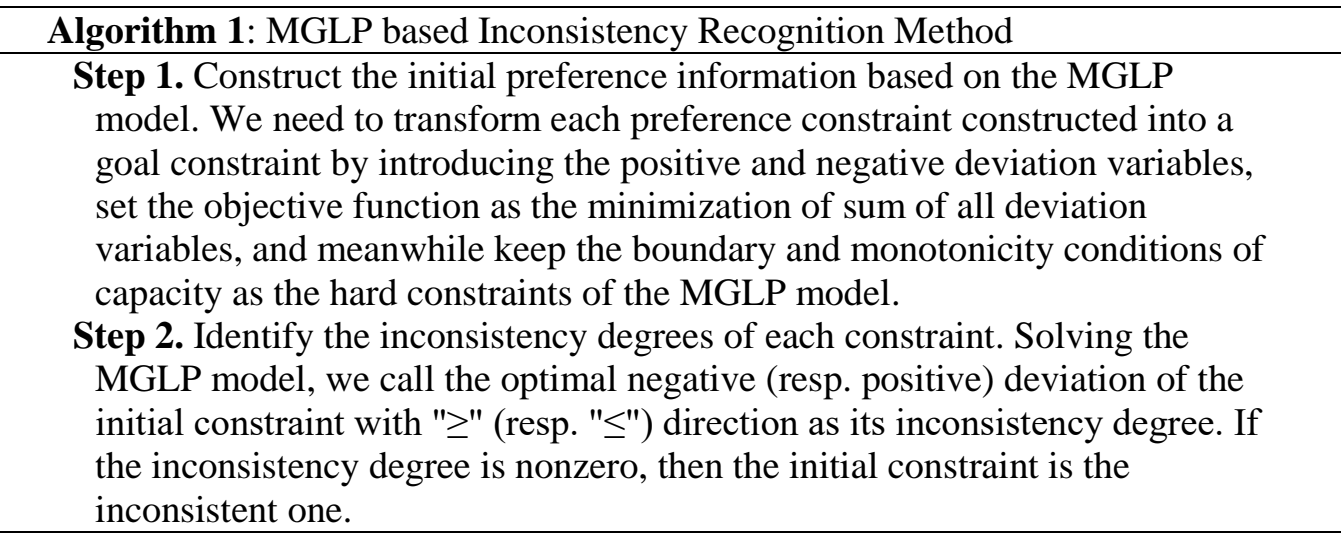

Remark 1. Suppose we have one preference constraint, $n_{\mu}(A)-n_{\mu}(B) \geq$ $(\leq) 0.1$, which means the nonadditivity index of subset $A$ is greater (less) than that of $B$ with threshold of 0.1 , we can introduce the positive and negative deviations variables, $d^{+}$and $d^{-}$, where $d^{+}, d^{-} \geq 0$ and $d^{+} \times d^{-}=0$ and get the goal constraint as

$$
n_{\mu}(A)-n_{\mu}(B)-d^{+}+d^{-}=0 .
$$

Furthermore, if the initial preference constraint's direction is "=", then we just represent this constraint into two constraints by respectively changing the direction as " $\geq$ " and " $\leq$ ". Generally, we can have the following MGLP model $[19,22]$ :

$$
\min \sum_{r=1}^{p} d_{r}^{+}+d_{r}^{-}
$$

s.t. the boundary and monotonicity of capacity, (3)

the goal constraints transformed from the preference information,

DOI: 10.24818/18423264/54.2.20.17 
Jian-Zhang Wu, Rui-JieXu, GlebBeliakov

where $d_{r}^{+}, d_{r}^{-} \geq 0, p$ is the amount of goal constraints in the above model. It should be mentioned that all the nonlinear constraints, $d_{r}^{+} \times d_{r}^{-}=0$, can be removed for the objective function is to minimize and the model is just a linear programming.

\subsection{Inconsistency adjustment strategies}

For the inconsistent preference constraint, we can just remove them from the constraint set or adjust them into consistent case. One inconsistency adjust strategy [19], called strategy S-I for convenience, is

S-I:to subtract the optimal negative deviation from (resp. plus the optimal positive deviation to) the right hand side of the inconsistent constraint if it is with " $\geq$ " (resp. " $\leq$ ") direction.

Alternatively, we can change the objective function of Model 3 by only keeping the positive and negative deviation variables of the inconsistent constraints (i.e., remove those variables of the consistent constraints) and meanwhile set the consistent constraints as strict equations (i.e., change all the directions of " $\geq$ " or " $\leq$ " into "="). One can figure out this changed model can get the same optimal solution of Model 3 because the minimum of the deviation of the consistent constraints are all zero, i.e., the consistent constraints are just strict equation with the optimal solutions of models 3 . That is Model 3 only check the boundary inconsistency case of the preference constraints.

Actually, we can further update the Model 3 to be more flexible and reasonable as follows:

$$
\min \sum_{r \in P_{\geq}} d_{r}^{-}+\sum_{r \in P_{\leq}} d_{r}^{+}
$$

s.t. the boundary and monotonicity of capacity,

the goal constraints transformed from the preference information, where $d_{r}^{+}, d_{r}^{-} \geq 0, r=1, \ldots, p, p$ is the amount of goal constraints, $P_{\geq}$and $P_{\leq}$are the number set of the preference goal constraints with the directions of $\geq$ and $\leq$, respectively. That is, we only concern the minimum of positive deviation variable $d^{-}$for the " $\geq$" type of constraint, and $d^{+}$for the " $\leq$" type of constraint. Obviously, if the minimum of the objective function in Model 4 is zero, then all the constraints are consistent.

Similarly, if get the optimal solutions of deviation variables, we can also change the objective function of Model 4 as only the sum of the positive and negative deviation variables of the inconsistent constraints and meanwhile set the consistent constraints as their initial states (not the goal constraints). This changed model still has same set of optimal solutions of initial model 4, which is also same to the domain of feasible capacities of S-I adjusted preference constraints.

The strategy S-I works in any situation even when no field knowledge are available and it also can be executed simultaneously to all the inconsistent 
Nonadditivity Index Oriented Decision Preference Information Representation and Capacity Identification

constraints $[19,22]$. However, if there is much available field knowledge of the applied indices or relatively clear estimation on the preference constraints, it is better to try some more flexible adjustment strategies.

Generally, from Eqs. (1) and (2), we can obtain that the nonadditivity index of a subset is in proportion to its capacity value and in inverse proportion to its proper subsets' capacity values and then in inverse proportion to its proper subsets' nonadditivity index values. That is, for a given $A \subseteq N$, if we aim to increase the value of $n_{\mu}(A)$, we can try to increase the value of $\mu(A)$ or decrease the values of $\mu(B), B \subset A$, or $n_{\mu}(B), B \subset A$ as well.Technically, we can

S-II: use the linear programming to help us obtain the range of an inconsistent constraint based on the consistent preference constraints and then according to the obtained range, some flexible adjustment strategies can be applied specifically.

Since the constraints in Models 3 and 4, including the boundary and monotonicity conditions and the decision maker's preference constraints, are all the linear ones, so the feasible range of any constraint must be a closed interval [2].

Furthermore, in models 3 and 4, its objective function is basically a weighted objective function where all the preference constraints are treated with the same weight 1 . In practice, it is possible for the decision maker to give his/her priority preference on those preference constraints. Suppose the preference constraints are divided into $m$ priority level sets: $\left\{P_{1}, P_{2}, \ldots, P_{m}\right\}$ in descending order, where the constraint in $P_{i}$ has absolutely higher priority than that in $P_{i+1}$. We can separately apply the inconsistency check and adjustment to each priority level set in sequence, or just adopt the following preemptive MGLP model to get the optimal capacities.

$$
\min \sum_{r \in P_{\geq}} K(r) d_{r}^{+}+\sum_{r \in P_{\leq}} K(r) d_{r}^{-}
$$

s.t. the boundary and monotonicity of capacity,

the goal constraints transformed from the preference information, where $P_{\geq}$and $P_{\leq}$are same as in Model $4, K(r)$ is the priority level function: $\{1, \ldots, p\} \rightarrow\left\{k_{1}, \ldots, k_{m}\right\}, m$ is the number of priority levels, and $k_{i}>>k_{i+1}, i=$ $1, \ldots, m-1$. The value of $k_{i}$ can be identified empirically in real problem by the decision maker or analyst. Then, based on the optimal deviation variables, we can adopt the strategies S-I or S-II to adjust the inconsistent constraint and get a feasible domain of compatible capacities.

\section{The nonadditivity indices oriented capacity identification method}

Inspired by the fact that the set of optimal capacities of MGLP model is essentially same as the feasible domain after applied adjustment strategy S-I as well as the study of the simultaneous interaction indices oriented capacity

DOI: 10.24818/18423264/54.2.20.17 
Jian-Zhang Wu, Rui-JieXu, GlebBeliakov

identification method [23], in this section we construct the nonadditivity index oriented MGLP model with less consistency check effort and manage to obtain the desired capacity that most close to the decision maker's preference expectation. That is, we construct the approach of using the coefficients of the selected deviation variables or equally the nonadditivity indices in the objective function of MGLP to present the preference information of decision maker on the importance or interaction of criteria.

As shown by the Model NSIIO in [23], if the objective is to maximize, the basic principle of identifying the coefficients is that the higher the preference constraint's expected value is, the greater its corresponding coefficient in objective function will be [23]. Similarly, we can construct the following Multiple Goal Nonlinear Programming.

$$
\begin{aligned}
& \operatorname{Max} z=\sum_{A \in K^{+}} w_{A} d_{A}^{+}+\sum_{A \in K^{-}} w_{A} d_{A}^{-}+\sum_{A \in K^{0}} w_{A}\left(d_{A}^{+}+d_{A}^{-}\right) \\
& \left\{\begin{array}{l}
\text { the boundary and monotonicity conditions, } \\
\text { the comparison form of perference constriants, } \\
n_{\mu}(A)-d_{A}^{+}+d_{A}^{-}=0, d_{A}^{+}, d_{A}^{-} \geq 0, d_{A}^{+} \times d_{A}^{-} \neq 0, A \in\left\{K^{+} \cup K^{-} \cup K^{0}\right\},
\end{array}\right.
\end{aligned}
$$

where $K^{+}, K^{-}, K^{0}$ are the sets of subsets whose nonadditivity indices are supposed to be positive, negative and zero, the coefficients $w_{A}$ according to the rule of [23], in objective function is set as $-9,-7,-5,-3,1,3,5,7$ and 9 if the nonadditivity index is expected to be extremely negative, strongly negative, fairly negative, slightly negative, zero, slightly positive, fairly positive, strongly positive and extremely positive respectively.

However, this model has some disadvantages. First, the model is non-linear model because the nonlinear constraints, $d_{A}^{+} \times d_{A}^{-} \neq 0$, can not be removed for the maximization objective function. Second, the coefficients are kind of fixed and too empirical to ensure the corresponding nonadditivity indices follow into their expected intervals like the 9-interval interaction scale talked in subsection 3.1. Third, the comparison form of constraints are still the hard constraints among which any inconsistency will lead to the infeasible solution for Model 6.

In the following, we update this model to a more flexible algorithm which is based on a minimization MGLP model and then manage to close the decision preference expectations of the nonadditivity indices to the maximum extent. The minimization MGLP model is given as:

$$
\begin{aligned}
& \text { Min } z=\sum_{A \in K^{0}}\left(d_{A}^{+}+d_{A}^{-}\right)+\sum_{A \in K^{+} \cup K^{-}} \lambda_{A} n_{\mu}(A) \\
& \left\{\begin{array}{l}
\text { the boundary and monotonicity conditions, } \\
\text { the comparison form of perference of singletons' nonadditivity indices, } \\
n_{\mu}(A)-d_{A}^{+}+d_{A}^{-}=0, d_{A}^{+}, d_{A}^{-} \geq 0, A \in K^{0},
\end{array}\right.
\end{aligned}
$$

where $K^{+}, K^{-}$and $K^{0}$ are same as Model $6, \lambda_{A}$ is set to be (called as Rule R-II)

$$
-1-9 \delta,-1-7 \delta,-1-5 \delta,-1-3 \delta, 1+3 \delta, 1+5 \delta, 1+7 \delta \text { and } 1+9 \delta
$$


Nonadditivity Index Oriented Decision Preference Information Representation and Capacity Identification

if the nonadditivity index of $A$ is supposed to be extremely positive, strongly positive, fairly positive, slightly positive, slightly negative, fairly negative, strongly negative and extremely negative respectively, where $\delta>0$ is a scale variable, see its possible range and purpose in Algorithm 2.

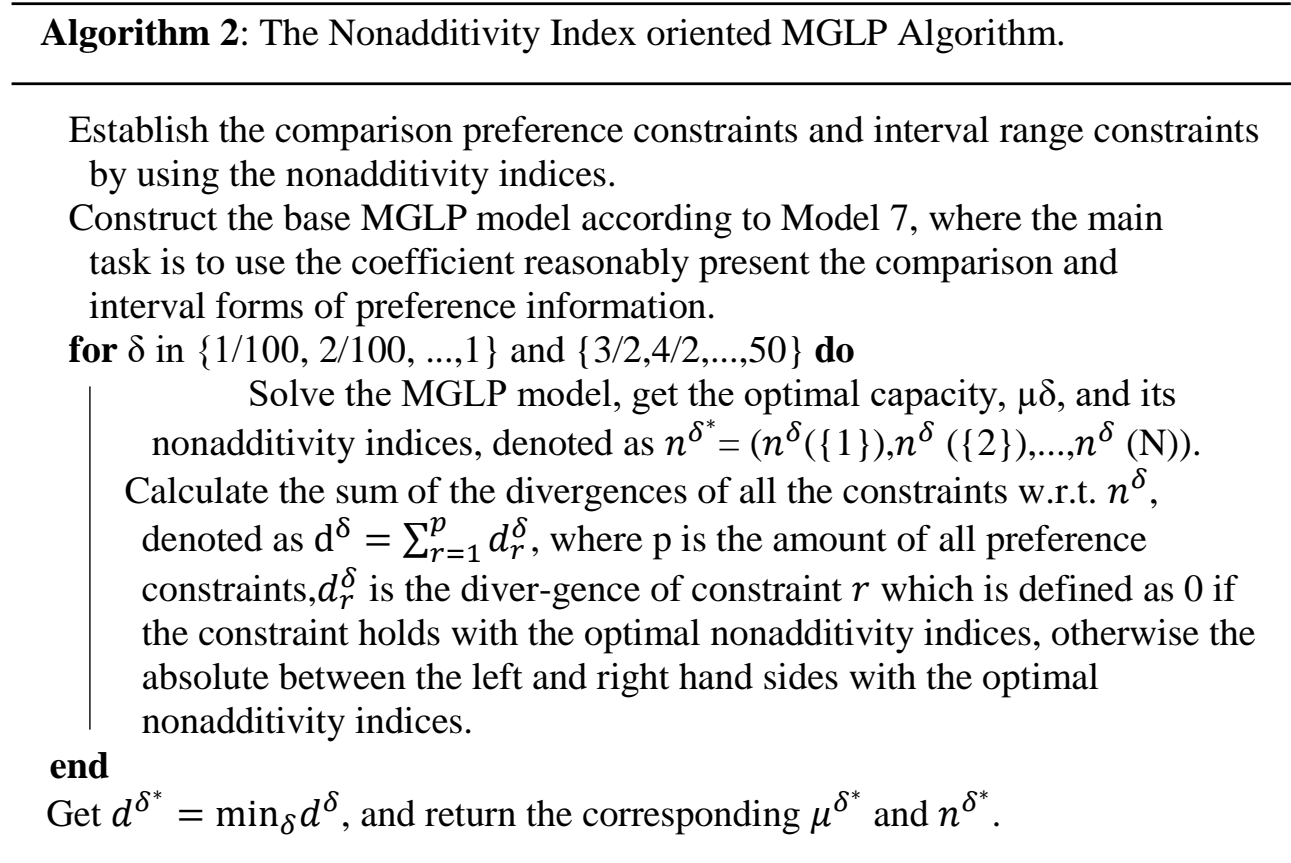

Remark 2. We should mention that we give the expected positive (negative) nonadditivity index a negative (positive) coefficient for the objective function of Model 7 is to minimize, and not to maximum as in Model 6.

Remark 3. One can notice that the Model 7 only includes the comparison constraints of singletons' nonadditivity indices, not for the comparison about higher order subsets. The main reason is, on the one hand there is a relatively large freedom for the singletons' indices to avoid inconsistency and it is also relatively easy to check and adjust if some inconsistency exists among them; on the other hand, we can transform the related comparison form constraints of higher order subsets into the range forms by virtue of the uniform range and good decision explanation of nonadditivity index, which can really reduce the inconsistency recognition effort exerting on the decision maker or analyst. For example, for the comparison form constraint: $n_{\mu}(B)-n_{\mu}(C) \geq 0.1$, there may be three cases of decision maker's judgment on the signs of $n_{\mu}(B)$ and $n_{\mu}(C)$ : (1) Both are positive, (2) Both are negative, and (3) $n_{\mu}(B)$ is positive and $n_{\mu}(C)$ is negative. For case (1) $n_{\mu}(B)$ and $n_{\mu}(C)$ can be assigned into different intervals, like $n_{\mu}(B)$ is strongly positive and $n_{\mu}(C)$ is fairly positive and they were given two different coefficients 
in objective function $-1-7 \delta$ and $-1-5 \delta$ respectively, or they are assigned in one same interval, e.g., both are fairly positive and in order to show their difference, we can give their coefficients as $-1-5 \delta$ and $-1-4 \delta$ respectively. Case (2) is similar to case (1). For case (3), we can assign $n_{\mu}(B)$ as slightly positive and $n_{\mu}(C)$ as slightly negative for threshold 0.1 is not relatively large compared to the range $[-1,1]$ of nonadditivity index, then their coefficients in objective function can be $-1-3 \delta$ and $1+3 \delta$.

Remark4. The coefficient $\lambda$ or equally $\delta$ are identified by the decision maker or analyst empirically, where the coefficients of zero additivity indices were taken as the base, 1 , and keep steady. And the $\min _{\delta} d^{\delta}$ in Algorithm 2 is to get the least sum of inconsistent deviations of all the range form constraints in Model 7, accordingly the $\mu^{\delta^{*}}$ orn ${ }^{\delta^{*}}$ is just the minimum deviation capacity or nonadditivity index that competently represents the decision maker's preference expectation.

\section{An Illustrative Example}

5.1 The inconsistency check and adjustment strategies

Suppose the decision problem involves five decision criteria, that is the criteria set is $\mathrm{N}=\{1,2,3,4,5\}$.

We begin with some simple comparison constraints to show and compare the different models and strategies. Assume the decision maker thinks that the importance of the five single criteria should be:

(1) criterion 1 is no less important that criterion 4;

(2) criterion 2 is more important than criterion 1 with threshold of 0.1 ;

(3) criterion 2 is more important than criterion 3 with threshold of 0.1 ;

(4) criterion 3 is no less important that criterion 5;

(5) criterion 4 is more important than criterion 5 with threshold of 0.1 .

If we describe the importance of criterion by its capacity value or equally the nonadditivity index, we can have:

$$
\begin{array}{ll}
n_{\mu}(\{1\})-n_{\mu}(\{4\}) & \geq 0 ; \\
n_{\mu}(\{2\})-n_{\mu}(\{1\}) & \geq 0.1 ; \\
n_{\mu}(\{2\})-n_{\mu}(\{3\}) & \geq 0.1 ;(8) \\
n_{\mu}(\{3\})-n_{\mu}(\{5\}) & \geq 0 ; \\
n_{\mu}(\{4\})-n_{\mu}(\{5\}) & \geq 0.1 .
\end{array}
$$

According to Model 3, we have 
Nonadditivity Index Oriented Decision Preference Information Representation and Capacity Identification

$$
\min \sum_{r=1}^{5} d_{r}^{+}+d_{r}^{-}
$$

s.t. the boundary and monotonicity of capacity,

$$
\begin{aligned}
& n_{\mu}(\{1\})-n_{\mu}(\{4\})-d_{1}^{+}+d_{1}^{-}=0, \\
& n_{\mu}(\{2\})-n_{\mu}(\{1\})-d_{2}^{+}+d_{2}^{-}=0.1, \\
& n_{\mu}(\{2\})-n_{\mu}(\{3\})-d_{3}^{+}+d_{3}^{-}=0.1, \\
& n_{\mu}(\{3\})-n_{\mu}(\{5\})-d_{4}^{+}+d_{4}^{-}=0, \\
& n_{\mu}(\{4\})-n_{\mu}(\{5\})-d_{5}^{+}+d_{5}^{-}=0.1,
\end{aligned}
$$

where $d_{r}^{+}, d_{r}^{-} \geq 0, r=1,2,3,4,5$. Solving this model, we have all the all the optimal solutions of deviation variables are zero except $d_{1}^{-*}=0.1$, which means the constraint $n_{\mu}(\{1\})-n_{\mu}(\{4\}) \geq 0$ is "inconsistent" in this context. According to the strategy S-I, we can subtract $d_{1}^{-*}$ from its right hand side and get the adjusted preference constraints as:

$$
\begin{aligned}
& n_{\mu}(\{1\})-n_{\mu}(\{4\}) \geq-\mathbf{0 . 1} ; \\
& n_{\mu}(\{2\})-n_{\mu}(\{1\}) \geq 0.1 ; \\
& n_{\mu}(\{2\})-n_{\mu}(\{3\}) \geq 0.1 ; \\
& n_{\mu}(\{3\})-n_{\mu}(\{5\}) \geq 0 ; \\
& n_{\mu}(\{4\})-n_{\mu}(\{5\}) \geq 0.1 .
\end{aligned}
$$

Basically, Eq. (9) is equal to the following MGLP:

$$
\min d_{1}^{+}+d_{1}^{-}
$$

s.t. the boundary and monotonicity of capacity,

$$
\begin{aligned}
& n_{\mu}(\{1\})-n_{\mu}(\{4\})-d_{1}^{+}+d_{1}^{-}=0, \\
& n_{\mu}(\{2\})-n_{\mu}(\{1\})=0.1, \\
& n_{\mu}(\{2\})-n_{\mu}(\{3\})=0.1, \\
& n_{\mu}(\{3\})-n_{\mu}(\{5\})=0, \\
& n_{\mu}(\{4\})-n_{\mu}(\{5\})=0.1,
\end{aligned}
$$

where $d_{r}^{+}, d_{r}^{-} \geq 0, r=1,2,3,4,5$. One can figure out that Eqs. (9) and (11) only check the inconsistency of the boundary case of Eq. (9) and lead to a unreasonable adjusted result, see Eq. (10).

Actually, the constraints in Eq. (8) are mutually compatible or Eq. (8) has a feasible domain of capacity. By using the updated model, Model 4, we can construct the following MGLP:

DOI: 10.24818/18423264/54.2.20.17 
Jian-Zhang Wu, Rui-JieXu, GlebBeliakov

$\min \sum_{r=1}^{5} d_{r}^{-}$

s.t. the constraints of Eq. (9)

Solving this MGLP, we have the optimal negative deviation variables are all zero, which means Eq.(8) is consistent. The Model (12) has multiple optimal solutions and the set of its optimal capacities is basically equal to the domain of feasible capacities of Eq. (8). But the Eq. (8) also has some redundant information. For example, one can figure out that for any feasible capacity, we can have $n_{\mu}(\{2\})-$ $n_{\mu}(\{3\}) \geq 0.2>0.1$, which means the third constraint in Eq. (8) has some redundancy. This redundancy can be reflected by one optimal solution of positive deviation variables of Model (12) where $d_{3}^{+*}=0.1$.

Now, we further add the following interval constraints:

$$
\begin{aligned}
n_{\mu}(\{1,2,3\}) & \geq 0.75 \\
n_{\mu}(\{1,2,3\}) & \leq 1 ; \\
n_{\mu}(\{1,2,4\}) & \geq 0.5 ; \\
n_{\mu}(\{1,2,4\}) & \leq 0.75 \\
n_{\mu}(\{2,3,4\}) & \geq 0.5 ; \\
n_{\mu}(\{2,3,4\}) & \leq 0.75 ; \\
n_{\mu}(\{1,2,3,4\}) & \geq-0.5 ; \\
n_{\mu}(\{1,2,3,4\}) & \leq-0.25 \\
n_{\mu}(\{2,3,4,5\}) & \geq-0.75 \\
n_{\mu}(\{2,3,4,5\}) & \leq-0.5 .
\end{aligned}
$$

That is, the interactions of $n_{\mu}(\{1,2,3\}), n_{\mu}(\{1,2,4\}), n_{\mu}(\{2,3,4\}), n_{\mu}(\{1,2,3,4\})$ and $n_{\mu}(\{2,3,4,5\})$ are expected to be extremely positive, strongly positive, strongly positive, fairly negative and strongly negative, respectively. According to Model 4, we can transform constraints of (8) and (13) into goal constraints and construct the following MGLP:

$$
\min \sum_{r \in P_{\geq}} d_{r}^{-}+\sum_{r \in P_{\leq}} d_{r}^{+}
$$

s.t. the boundary and monotonicity of capacity,

$$
\begin{aligned}
& n_{\mu}(\{1\})-n_{\mu}(\{4\})-d_{1}^{+}+d_{1}^{-}=0, \\
& \ldots \\
& n_{\mu}(\{1,2,3\})-d_{6}^{+}+d_{6}^{-}=0.75, \\
& \quad \ldots \\
& n_{\mu}(\{2,3,4,5\})-d_{15}^{+}+d_{15}^{-}=-0.5,
\end{aligned}
$$

where $P_{\geq}=\{1,2,3,4,5,6,8,10,12,14\}, P_{\leq}=\{7,9,11,13,15\}$. Solving this model, we have the optimal objective function value is 0.6 , which means there are some inconsistencies among the constraints. More specifically, $d_{5}^{-*}=0.05$ for 
Nonadditivity Index Oriented Decision Preference Information Representation and Capacity Identification

constraints whose indices are in $P_{\geq}$and $d_{13}^{+*}=0.2642858, d_{15}^{+*}=0.2857143$ for constraints whose indices are in $P_{\leq}$, which means the constraints 5, 13 and 15 are inconsistent. By adopting the strategy S-I, we can adjust these three initial constraints as:

$$
\begin{aligned}
& n_{\mu}(\{4\})-n_{\mu}(\{5\}) \geq 0.1-\mathbf{0 . 0 5}=\mathbf{0 . 0 5}, \\
& n_{\mu}(\{1,2,3,4\}) \leq 0.25+\mathbf{0 . 2 6 4 2 8 5 8}=\mathbf{0 . 0 1 4 2 8 5 8} \\
& n_{\mu}(\{2,3,4,5\}) \leq 0.5+\mathbf{0 . 2 8 5 7 1 4 3}=-\mathbf{0 . 2 1 4 2 8 5 7}
\end{aligned}
$$

Then we use the Model 4 to check again and get that all the constraints are consistent. We'd like to mention that the set of optimal capacities of Eq. (14) is same to the domain of feasible capacities given by the adjusted constraints and also same to set of optimal capacities of MGLP model with adjusted constraints.

We illustrate the strategy S-II for Eq. (14). Since Eq. (8) is consistent, we can first check minimum and maximum of $n_{\mu}(\{1,2,3,4\})$ by using the following linear programming:

min or max $n_{\mu}(\{1,2,3,4\})$

s.t. the boundary and monotonicity of capacity,

Eqs.(8) and (13) (meanwhile remove the last four constraints,

which involve $n_{\mu}(\{1,2,3,4\})$ and $\left.n_{\mu}(\{2,3,4,5\})\right)$.

Solving the above linear programming, we have $\min n_{\mu}(\{1,2,3,4\})=$ 0.01428571 and $\max n_{\mu}(\{1,2,3,4\})=0.5404762$. Suppose the decision maker, in view of this, determine to adjust the interaction of $\{1,2,3,4\}$ as slightly positive, i.e., $n_{\mu}(\{1,2,3,4\}) \geq 0$ and $n_{\mu}(\{1,2,3,4\}) \leq 0.25$. Further taking account of these above two constraints, we can get the minimum and maximum of $\{2,3,4,5\}$ are 0.2190476 and 0.7452381 respectively. Then decision maker may adjust the interaction of $\{2,3,4,5\}$ as slightly negative, i.e., $n_{\mu}(\{2,3,4,5\}) \geq-0.25$ and $n_{\mu}(\{2,3,4,5\}) \leq 0$.

We can further try the preemptive MGLP model. Suppose the decision maker think the constraints in Eq. (8) shouldhave the $P_{2}$ level priority. According to Model (5), we can set $K(r)=k_{1}$ for $r=1, \ldots 5$ and $K(r)=k_{2}$ for $r=6, \ldots 15$. If set $k_{1}=100$ and $k_{2}=1$, we can get the following optimal deviation variables are not zero: $d_{6}^{-*}=0.01666667$ for constraints whose indices are in $P_{\geq}$and $d_{13}^{+*}=$ $0.30714286, d_{15}^{+*}=0.28571429$ for constraints whose indices are in $P_{\geq}$, which means the constraints 6,13 and 15 are inconsistent. Actually, if we decrease $k_{1}$ from 100 to 2 and keep $k_{2}$ as 1 , we can also get the above result of inconsistency. If we further decrease the value of $k_{1}$, the inconsistency case can change, e.g., if $k_{1}=1.9$, the result is $d_{5}^{-*}=0.01, d_{13}^{+*}=0.3042857$ and $d_{15}^{+*}=0.2857143$, which is a similar case of Model 14.

DOI: 10.24818/18423264/54.2.20.17 
Jian-Zhang Wu, Rui-JieXu, GlebBeliakov

5.2 The nonadditivity index oriented algorithm

Now besides the constraints Eqs. (8) and (13), we further add the following preference constraints:

$$
\begin{aligned}
& n_{\mu}(\{2,3\})=0 \\
& n_{\mu}(\{1,3,4\})=0 \\
& n_{\mu}(\{1,2,4,5\}) \geq-0.25 \\
& n_{\mu}(\{1,2,4,5\}) \leq 0 \\
& n_{\mu}(\{1,2,3,4,5\}) \geq-0.5 \\
& n_{\mu}(\{1,2,3,4,5\}) \leq-0.25
\end{aligned}
$$

Then according to Model 7,for we can get the following MGLP:

$$
\begin{aligned}
& \min z=d_{1}^{+}, d_{1}^{-}, d_{2}^{+}, d_{2}^{-}+(-1-9 \delta) n_{\mu}(\{1,2,3\}) \\
& +(-1-7 \delta) n_{\mu}(\{2,3,4\})+(-1-7 \delta) n_{\mu}(\{1,2,4\}) \\
& +(1+5 \delta) n_{\mu}(\{1,2,3,4\})+(1+7 \delta) n_{\mu}(\{2,3,4,5\}) \\
& +(1+3 \delta) n_{\mu}\left(\{1,2,4,5\}+(1+5 \delta) n_{\mu}(\{1,2,3,4,5\}\right. \\
& \text { s.t. }\left\{\begin{array}{l}
\text { the boundary and monotonicity conditions, } \\
\text { Eq. }(8), \\
n_{\mu}(\{2,3\})-d_{1}^{+}+d_{1}^{-}=0, \\
n_{\mu}(\{1,3,4\})-d_{2}^{+}+d_{2}^{-}=0,
\end{array}\right.
\end{aligned}
$$

where $d_{1}^{+}, d_{1}^{-}, d_{2}^{+}, d_{2}^{-} \geq 0$.

By executing the Algorithm 2, we have the sums of optimal inconsistent deviations, as shown in Table 1 . One can figure out that $d^{\delta^{*}}=\min _{\delta} d^{\delta}=$ 0.8366667 , where $\delta^{*}=1 / 100 \sim 6 / 100$. Their corresponding nonadditivity index and capacity values are listed in Tables 2 and 3. From the former table we can see the nonadditivity indices are relatively consistent with the decision preference; in the latter table, the capacity values show more extremely trend, e.g., almost the 3 or higher order subsets' capacities are 1, which mainly because in the one hand the nonadditivity indices of Eq. (8) are required as extremely positive or strongly negative, on the other hand, nonadditivity index oriented model has kind of trend to obtain the extreme solution, because of the fact of relatively less number of the hard constraints. 
Nonadditivity Index Oriented Decision Preference Information Representation and Capacity Identification

Table 1: The values of $\delta$ and the corresponding sum of deviations

\begin{tabular}{clllll}
\hline$\delta$ & $1 / 100 \sim 6 / 100$ & $7 / 100 \sim 28 / 100$ & $29 / 100 \sim 57 / 100$ & $58 / 100 \sim 1$ & $3 / 2 \sim 50$ \\
\hline $\mathrm{d}^{\delta}$ & 0.8366667 & 0.9095238 & 0.9809524 & 1.2380952 & 1.7285714 \\
\hline
\end{tabular}

Table 2: The optimal nonadditivity index on criteria set $\{1,2,3,4,5\}$

\begin{tabular}{cccccccc}
\hline$A$ & $n_{\mu}(A)$ & $A$ & $n_{\mu}(A)$ & $A$ & $n_{\mu}(A)$ & $A$ & $n_{\mu}(A)$ \\
\hline$\varnothing$ & 0 & $\{1,4\}$ & 0.1 & $\{1,2,3\}$ & 0.7333 & $\{2,4,5\}$ & 0.1667 \\
$\{1\}$ & 0.1 & $\{1,5\}$ & 0.9 & $\{1,2,4\}$ & 0.6333 & $\{3,4,5\}$ & 0.2 \\
$\{2\}$ & 0.2 & $\{2,3\}$ & 0.0001 & $\{1,2,5\}$ & 0.1667 & $\{1,2,3,4\}$ & 0.2857 \\
$\{3\}$ & 0 & $\{2,4\}$ & -0.1 & $\{1,3,4\}$ & 0.0001 & $\{1,2,3,5\}$ & -0.0286 \\
$\{4\}$ & 0.1 & $\{2,5\}$ & 0.8 & $\{1,3,5\}$ & 0.2667 & $\{1,2,4,5\}$ & -0.1571 \\
$\{5\}$ & 0 & $\{3,4\}$ & 0.2 & $\{1,4,5\}$ & 0.1667 & $\{1,3,4,5\}$ & -0.0286 \\
$\{1,2\}$ & -0.1 & $\{3,5\}$ & 1 & $\{2,3,4\}$ & 0.6667 & $\{2,3,4,5\}$ & -0.1429 \\
$\{1,3\}$ & 0.0001 & $\{4,5\}$ & 0.9 & $\{2,3,5\}$ & 0.2 & $\{1,2,3,4,5\}$ & -0.3333 \\
\hline
\end{tabular}

Table 3: The optimal capacity on criteria set $\{1,2,3,4,5\}$

\begin{tabular}{cccccccc}
\hline$A$ & $\mu(A)$ & $A$ & $\mu(A)$ & $A$ & $\mu(A)$ & $A$ & $\mu(A)$ \\
\hline$\varnothing$ & 0 & $\{1,4\}$ & 0.3 & $\{1,2,3\}$ & 1 & $\{2,4,5\}$ & 1 \\
$\{1\}$ & 0.1 & $\{1,5\}$ & 1 & $\{1,2,4\}$ & 1 & $\{3,4,5\}$ & 1 \\
$\{2\}$ & 0.2 & $\{2,3\}$ & 0.2 & $\{1,2,5\}$ & 1 & $\{1,2,3,4\}$ & 1 \\
$\{3\}$ & 0 & $\{2,4\}$ & 0.2 & $\{1,3,4\}$ & 1 & $\{1,2,3,5\}$ & 1 \\
$\{4\}$ & 0.1 & $\{2,5\}$ & 0.3 & $\{1,3,5\}$ & 0.2667 & $\{1,2,4,5\}$ & 1 \\
$\{5\}$ & 0 & $\{3,4\}$ & 0.3 & $\{1,4,5\}$ & 1 & $\{1,3,4,5\}$ & 1 \\
$\{1,2\}$ & 0.2 & $\{3,5\}$ & 1 & $\{2,3,4\}$ & 1 & $\{2,3,4,5\}$ & 1 \\
$\{1,3\}$ & 0.1 & $\{4,5\}$ & 1 & $\{2,3,5\}$ & 1 & $\{1,2,3,4,5\}$ & 1 \\
\hline
\end{tabular}

\section{Conclusions}

The nonadditivity index has some relatively good mathematical characteristics and a fairly suitable explanation in decision making context, which make it more reasonable to directly compare the interactions with different orders' subsets and to easily arrange the interaction density into regular ranges. The updated inconsistency recognition MGLP models and adjustment strategies help to represent the decision maker's preference information more correctly and efficiently. Furthermore, the nonadditivity index oriented capacity identification method can find out some capacities that rather close the decision maker's preference with relatively less efforts on the inconsistency checking and adjustment. The inconsistency recognition and capacity identification methods and strategies discussed in this paper can provide a useful reference for representing the decision maker's preference information by other representations of capacity, like the bipartition interaction index and nonmodularity index. The further research task can focus on the application of these methods in real decision problems as well as the empirical comparison analysis of difference representations of capacity in depicting the same preference information.

DOI: $10.24818 / 18423264 / 54.2 .20 .17$ 
Jian-Zhang Wu, Rui-JieXu, GlebBeliakov

\section{ACKNOWLEDGEMENTS}

The work was supported by the National Natural Science Foundation of China (No.71671096) and the K.C.Wong Magna Fund in Ningbo University.

\section{REFERENCES}

[1] Beliakov, G. (2009), Construction of Aggregation Functions from Data Using Linear Programming. Fuzzy Sets and Systems, 160(1):65-75;

[2]Beliakov,G., S. James, and J.-Z. Wu(2019),Discrete Fuzzy Measures:

Computational Aspects. Springer, Cham, Switzerland;

[3]Beliakov, G., H. B. Sola, and T. Calvo (2016), A Practical Guide to

Averaging Functions. Springer, New York;

[4]Chateauneuf, A. and J.-Y. Jaffray (1989), Some Characterizations of Lower

Probabilities and other Monotone Capacities through the Use of Möbius

Inversion.Mathematical social sciences, 17(3):263-283;

[5]G. Choquet (1954), Theory of Capacities. Annales de l'institut Fourier, 5:131295 ;

[6]S. Corrente, S. Greco, and A. Ishizaka(2016), Combining Analytical Hierarchy Process and Choquet Integral within Non-additive Robust Ordinal Regression.Omega, 61:2-18;

[7]S.Corrente, S. Greco, M. Kadziński and R. Słowiński(2013), Robust Ordinal Regression in Preference Learning and Ranking.Machine Learning, 93(23):381422;

[8]K. Fujimoto(2010), Representations of Importance and Interaction of Fuzzy

Measures, Capacities, Games and its Extensions: A Survey.In Integrated

Uncertainty Management and Applications, pages 115-126. Springer, Berlin, New York;

[9]K. Fujimoto, I. Kojadinovic and J.-L. Marichal(2006), Axiomatic

Characterizations of Probabilistic and Cardinal-Probabilistic Interaction Indices. Games and Economic Behavior, 55(1):72-99;

[10]M. Gagolewski, S. James and G. Beliakov(2019),Supervised Learning to Aggregate Data with the Sugeno Integral.IEEE Transactions on Fuzzy Systems; [11]M.Grabisch(1997),k-Order Additive Discrete Fuzzy Measures and their Representation.Fuzzy Sets and Systems, 92(2):167-189;

[12]M. Grabisch, I. Kojadinovic, and P. Meyer(2008), A Review of Methods for Capacity Identification in ChoquetIntegral Based Multi-Attribute Utility Theory: Applications of the Kappalab R Package.European Journal of Operations Research, 186(2):766-785;

[13]M. Grabisch and C. Labreuche(2010), A Decade of Application of the

Choquetand SugenoIntegrals in Multi-Criteria Decision Aid. Annals of

Operations Research, 175(1):247-286; 
Nonadditivity Index Oriented Decision Preference Information Representation and Capacity Identification

[14]M. Grabisch and M. Roubens(1999), Probabilistic Interactions among Players of a Cooperative Game.In M. Machina and B. Munier, editors, Beliefs, Interactions and Preferences in Decision Making, pages 205-216. Springer,

Heidelberg, New York;

[15]E. Pap(1995), Null-additive Set Functions. Kluwer Academic Pub, Dordrecht; [16]M. Ridaoui, M. Grabisch and C. Labreuche(2019), Interaction Indices for Multichoice Games. Fuzzy Sets and Systems;

[17]M. Sugeno(1974), Theory of Fuzzy Integrals and its Applications. PhD thesis, Tokyo Institute of Technology;

[18]J.-Z. Wu and G. Beliakov(2018),Nonadditivity Index and Capacity Identification Method in the Context of Multicriteria Decision Making. Information Sciences, 467:398-406;

[19]J.-Z. Wu and G. Beliakov(2019),Nonadditive Robust Ordinal Regression with Nonadditivity Index and Multiple Goal Linear Programming. International Journal of Intelligent Systems, 34(7):1732-1752;

[20]J.-Z. Wu and G. Beliakov(2019), Nonmodularity Index for Capacity Identifying with Multiple Criteria Preference Information.Information Sciences, 492:164-180;

[21]J.-Z. Wu and G. Beliakov(2019), Probabilistic Bipartition Interaction Index of Multiple Decision Criteria Associated with the Nonadditivity of Fuzzy

Measures. International Journal of Intelligent Systems, 34(2):247-270; [22]J.-Z. Wu, L. Huang, R.-J. Xi and Y.-P. Zhou(2019), Multiple Goal Linear Programming Based Decision Preference Inconsistency Recognition and Adjustment Strategies. Information, 10(7):223;

[23]J.-Z. Wu, E. Pap and A. Szakal(2018),Two Kinds of Explicit Preference Information Oriented Capacity Identification Methods in the Context of Multicriteria Decision Analysis. International Transactions in Operational Research, 25:807-830;

[24]J.-Z. Wu, S. Yang, Q. Zhang and S. Ding(2015), 2-Additive Capacity Identification Methods from Multicriteria Correlation Preference Information.IEEE Transactions on Fuzzy Systems, 23(6):2094-2106;

[25]J.-Z. Wu, Q. Zhang, Q. Du and Z. Dong(2014), Compromise Principle Based Methods of Identifying Capacities in the Framework of Multicriteria Decision Analysis. Fuzzy Sets and Systems, 246:91-106.

DOI: $10.24818 / 18423264 / 54.2 .20 .17$ 